\title{
Nuestra experiencia con el catéter autoposicionante frente a otras variantes del catéter Tenckhoff. Estudio multicéntrico
}

\author{
Francisco Cirera Segura*, Jesús Lucas Martín Espejo*, Antonia Concepción Gómez Castilla**, \\ $M^{a}$ Ángeles 0 jeda Guerrero**
}

Enfermeros/as. * Unidad de Gestión Clínica Uro-Nefrológica. H.U. Virgen del Rocío

** Unidad de Gestión Clínica Nefrológica. H.U. Virgen Macarena. Sevilla

\section{Resumen}

\section{Introducción}

El objetivo del estudio fue comparar las complicaciones quirúrgicas, mecánicas e infecciosas que presenta el catéter autoposicionante frente a otros tipos de catéteres tipo Tenckhoff, así como comparar la supervivencia de ambos tipos de catéteres.

\section{Material y métodos}

Estudio descriptivo retrospectivo multicéntrico, de 79 meses de duración. Se estudiaron todos los catéteres colocados en dos unidades de diálisis peritoneal. El análisis se realizó con el paquete estadístico IBM SPSS Statistics 19.0, estableciendo el nivel de significación en $p<0,05$.

\section{Resultados}

La muestra la compusieron 241 catéteres en 202 pacientes, el $52,5 \%$ eran hombres $(n=106)$. La edad media fue $61,98+15,87$ años. El catéter más utilizado fue el autoposicionante 63,5\% ( $n=153)$.

Complicaciones Quirúrgicas. Solo se describieron en el $28,1 \%$ de los autoposicionante $(p<0,001)$.

Correspondencia:

Francisco Cirera Segura

Calle Ángel Ripoll Pastor, 4-6 $\mathrm{A}$

41006. Sevilla

e-mail: paco.cirera@gmail.com
Complicaciones Mecánicas. Fueron más frecuentes en el catéter autoposicionante excepto el desplazamiento, sólo fueron significativas para las fugas $(p=0,003)$.

Infecciones. Tuvieron lugar un total de 196 infecciones peritoneales en 116 catéteres, con una tasa de $0,691+0,87$ episodio por paciente y año. El $45,1 \%$ tuvo lugar en catéteres autoposicionantes, sin diferencias significativas $(p=0,214)$.

Causas de retirada. La salida de DP supuso un $45,6 \%$ de las retiradas de catéter, el $10 \%$ por infección peritoneal y el $6,6 \%$ por mal funcionamiento. El autoposicionante se retiró en más casos por fuga, infecciones peritoneales y mal funcionamiento.

Supervivencia de los catéteres. No hallamos diferencias en la supervivencias de los catéteres (Log-Rank Mantel Cox $=0,164$ ).

\section{Discusión}

Los catéteres autoposicionantes tuvieron un mayor número de complicaciones quirúrgicas y mecánicas que los Tenckhoff, sin encontrar diferencias respecto a las complicaciones infecciosas.

Hallamos una menor supervivencia respecto a los Tenckhoff sin significación estadística.

Todos los datos parecen relacionarse con las fugas producidas por los catéteres autoposicionantes desde su implantación. 
PALABRAS CLAVE:

- CATÉTER AUTOPOSICIONANTE

- CATÉTER TENCKHOFF

- DIÁLISIS PERITONEAL

\section{Our experience with the self-locating catheter compared to other variants of the Tenckhoff catheter. Multicentre study}

\section{Abstract}

\section{Introduction}

The aim of the study was to compare the surgical, mechanical and infectious complications presented by self-locating catheters compared to other types of Tenckhoff catheters, and to compare the survival of both types of catheter.

\section{Material and methods}

Multicentre retrospective descriptive study lasting 79 months. All the catheters placed in two peritoneal dialysis units were studied. The analysis was carried out using the IBM SPSS Statistics 19.0 statistical package, setting the significance level at $p<0.05$.

\section{Results}

The sample was made up of 241 catheters in 202 patients, of whom $52.5 \%$ were men $(n=106)$. The average age was $61.98+15.87$ years. The most used catheter was the self-locating catheter with $63.5 \%(n=153)$.

Surgical complications. Surgical complications were only described in $28.1 \%$ of the self-locating catheters $(p<0.001)$.

Mechanical complications. These were more frequent with the self-locating catheter, except for displacement, and were only significant for leaks $(p=0.003)$.

Infections. A total of 196 peritoneal infections took place in 116 catheters, with a rate of $0.691+$ 0.87 episode per patient per year. Of these, $45.1 \%$ occurred in self-locating catheters, with no significant differences $(p=0.214)$.
Causes for withdrawal. Leaving PD represented $45.6 \%$ of the catheter withdrawals, $10 \%$ were due to peritoneal infection and $6.6 \%$ due to incorrect function. The self-locating catheter was removed in more cases due to leaks, peritoneal infections and incorrect function.

Survival of the catheters. We did not find differences in the survival of the catheters (Log-Rank Mantel Cox $=0,164$ ).

\section{Discussion}

Self-locating catheters had a higher number of surgical and mechanical complications than the Tenckhoff catheters, with no differences found in respect of infectious complications.

We found a lower survival compared to the Tenckhoff catheters without statistical significance.

All the data seem to be related with the leaks caused by self-locating catheters since they were placed.

\section{KEY WORDS}

- SELF-LOCATING CATHETER

- TENCKHOFF CATHETER

- PERITONEAL DIALYSIS

\section{Introducción}

Uno de los principales problemas que ha tenido la Diálisis Peritoneal (DP) para su desarrollo ha sido el no poder contar con un acceso seguro al peritoneo, hasta que Tenckhoff diseñó su catéter peritoneal ${ }^{1}$. A este catéter se le han realizado múltiples variaciones buscando mejorar su rendimiento y disminuir el índice de complicaciones que presentan, como el desplazamiento de la punta, su atrapamiento por el epiplón o las diferentes complicaciones infecciosas. El catéter autoposicionante diseñado por Di Paolo ${ }^{2}$ tiene como como característica propia disponer de un peso de tungsteno (12 gramos) en su punta distal para evitar las complicaciones antes mencionadas.

El objetivo principal de nuestro estudio fue comparar las complicaciones quirúrgicas, mecánicas e infecciosas 
que presenta el catéter autoposicionante frente a otros tipos de catéteres tipo Tenckhoff.

Los objetivos secundarios fueron comparar la supervivencia de ambos tipos de catéteres así como analizar las variables que pudieran influir sobre la misma.

\section{Material y métodos}

Se realizó un estudio descriptivo retrospectivo multicéntrico, de 79 meses de duración del 24 de mayo de 2005 a 31 de diciembre de 2011 . Se estudiaron todos los catéteres colocados en las dos unidades de DP de referencia de nuestra ciudad. En una de ellas se utilizaba el catéter autoposicionante mientras que en la otra se utilizaban catéteres basados en el diseño de Tenckhoff con varias modificaciones: 2 cuff y cola de cerdo o 2 cuff y cuello de cisne.

Se recogieron datos demográficos y epidemiológicos, datos relacionados con la implantación, uso y retirada del catéter, así como los relacionados con la técnica y la salida de DP. También se recogieron complicaciones relacionadas con la técnica de implantación del catéter, fugas, hernias, extrusión del cuff externo, etc. así como los datos relacionados con las infecciones peritoneales (IP), del orificio de salida (IOS) y del túnel subcutáneo (ITS). Se descartaron las recidivas de las infecciones para el estudio.

Para realizar la estadística descriptiva de las variables del estudio usamos frecuencias absolutas y relativas en el caso de las variables cualitativas. Las cuantitativas según si su distribución fuera normal o no, se resumieron mediante la media y la desviación estándar o la mediana y el rango intercuartílico respectivamente. Realizamos la comprobación de los grupos del estudio mediante el test de Chi cuadrado o el test exacto de Fisher según correspondiese. El estudio de supervivencia se realizó mediante curvas de Kaplan-Meier y se compararon con el test de Log-Rank (Mantel-Cox). Para el análisis multivariante se utilizó la regresión de Cox donde se introdujeron como variables la edad, la fuga y las infecciones peritoneales.

El análisis se realizó con el paquete estadístico IBM SPSS Statistics 19.0, estableciendo el nivel de significación en $p<0,05$.

\section{Resultados}

La muestra la compusieron un total de 241 catéteres en 202 pacientes, el 52,5\% eran hombres $(n=106)$. La edad media fue $61,98+15,87$ años. El 45,5\% ( $n=92)$ estaban en diálisis peritoneal continua ambulatoria y $54,5 \%(n=110)$ en diálisis peritoneal automática. La etiología de la Enfermedad Renal Crónica se muestra en la Tabla 1.

\begin{tabular}{|l|c|}
\hline No filiada & $\begin{array}{c}\text { Porcentaje válido } \\
\text { (Frecuencia) }\end{array}$ \\
\hline Nefropatía Diabética & $34,2 \%(n=69)$ \\
\hline Glomerulonefritis & $16,8 \%(n=34)$ \\
\hline Enfermedades Vasculares & $15,8 \%(n=32)$ \\
\hline Pielonefritis/Nefritis Intersticial & $12,4 \%(n=25)$ \\
\hline Enfermedades Sistémicas & $11,4 \%(n=23)$ \\
\hline Enfermedad Poliquística & $5,4 \%(n=11)$ \\
\hline Enfermedades Hereditarias/Congénitas & $\mathbf{2 , 5 \% ( n = 5 )}$ \\
\hline & $1,5 \%(n=3)$ \\
\hline *Códigos EDTA 1994-1995 y su agrupación, extraídos del \\
Informe 2006. Módulo Básico. Subsistema de Insuficiencia Renal \\
Crónica. Servicio Andaluz de Salud. Junta de Andalucía.
\end{tabular}

Tabla 1. Etiología de la Enfermedad Renal Crónica*

El catéter más utilizado fue el autoposicionante $63,5 \%$ $(n=153)$, frente al $31,5 \% \quad(n=76)$ del catéter con cuello de cisne, y $5 \%$ de catéteres con cola de cerdo $(n=12)$.

\section{Complicaciones Quirúrgicas}

Cuando analizamos los problemas quirúrgicos acontecidos durante la colocación del catéter, sólo se describieron en el $28,1 \% \quad(n=43)$ de los catéteres autoposicionante no hallándose en ningún caso con los catéteres Tenckhoff, con la consecuente significación estadística en la comparativa entre los catéteres $(p<0,001)$. Se analizó si aquellos catéteres que habían tenido complicaciones quirúrgicas presentaron más complicaciones mecánicas y solo fue significativo en el caso de la fuga $(p<0,001)$.

\section{Complicaciones Mecánicas}

En la tabla 2 se recogen las complicaciones mecánicas que presentaron ambos tipos de catéteres. 
Todas fueron más frecuentes en el catéter autoposicionante excepto el desplazamiento, aunque sólo se encontraron diferencias significativas para las fugas $(p=0,003)$.

Al analizar las complicaciones que se presentaron cuando se utilizaron los catéteres antes del mes, sólo se encontraron diferencias significativas para el atrapamiento $(p=0,028)$.

\begin{tabular}{|c|c|c|c|}
\hline & \multicolumn{2}{|c|}{ Tipo de Catéter } & \multirow[t]{2}{*}{$\mathbf{p}^{1}$} \\
\hline & $\begin{array}{l}\text { Catéteres } \\
\text { Tenckhoff }\end{array}$ & $\begin{array}{c}\text { Catéteres } \\
\text { Autoposicionantes }\end{array}$ & \\
\hline Atrapamiento & $5,7 \%(n=5)$ & $7,2 \%(n=11)$ & 651 \\
\hline Extrusión & $13,6 \%(n=12)$ & $15,7 \%(n=24)$ & 667 \\
\hline Fuga & $0 \%$ & $11,8 \%(n=18)$ & ,001 \\
\hline Hernia & $6,8 \%(n=6)$ & $9,8 \%(n=15)$ &, 429 \\
\hline Desplazamiento & $16,1 \%(n=14)$ & $11,1 \%(n=17)$ & ,269 \\
\hline
\end{tabular}

Tabla 2. Complicaciones mecánicas en Diálisis Peritoneal

\section{Infecciones}

Tuvieron lugar un total de 196 IP en 116 catéteres, con una tasa de 0,691+0,87 episodio por paciente y año. De los 116 catéteres que presentaron IP, el $45,1 \%(n=69)$ eran autoposicionantes frente a los Tenckhoff, con un 53,4\% ( $n=47)$, no encontrándose diferencias significativas entre los grupo $(p=0,214)$.

En la Tabla 3 se presenta el análisis de los microrganismos causantes de las IP, de las IOS y de las ITS.

Los microrganismos más frecuente en las IP fueron los Gram $+, 61,74 \%(n=121)$. Se estudiaron por separado los estafilococos aureus por sus importancia y repercusión en DP. En las IP los estafilococos aureus metil-sensibles supusieron el $15,31 \%(n=30)$, del orificio el $39,78 \%(n=37)$ y en el túnel subcutáneo el $60 \%(n=18)$. Los estafilococos aureus metil-resistentes aparecieron en porcentajes menores, respectivamente en $1,02 \%(n=2), 2,15 \%(n=2)$ y $3,33 \%(n=1)$.

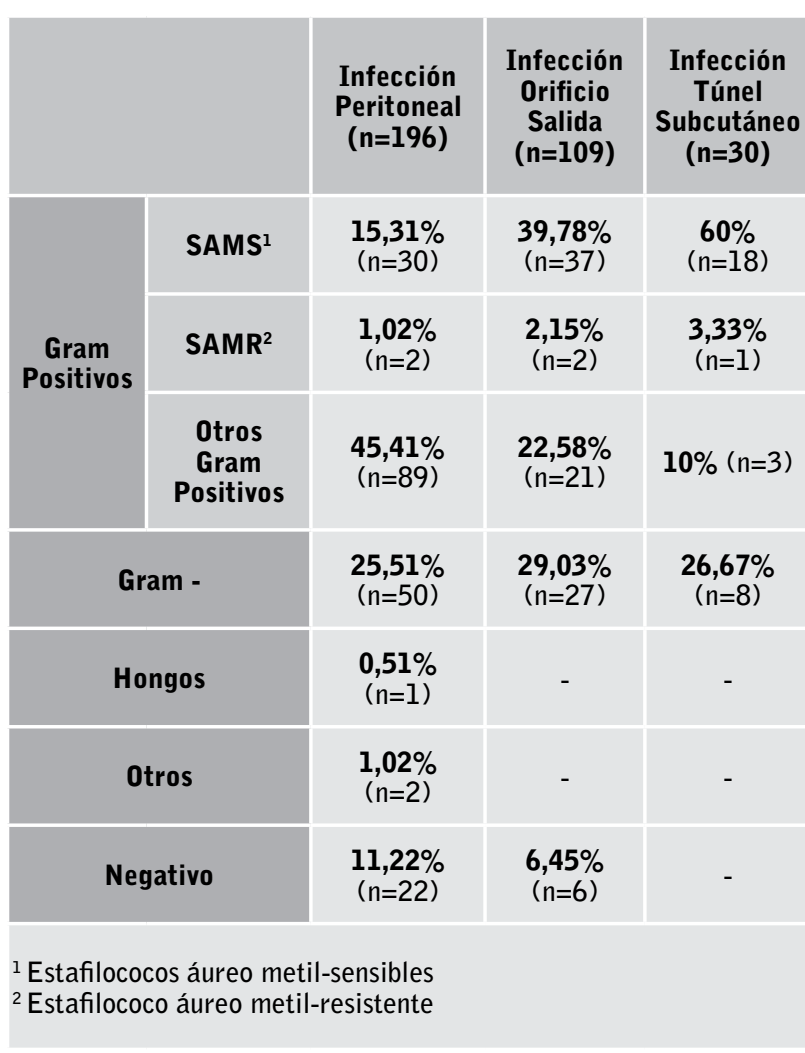

Tabla 3. Infecciones Peritoneales, del Orificio de Salida y del Túnel Subcutáneo

Se detectaron 58 portadores nasales de estafilococos aureus $(28,7 \%)$. De los 88 pacientes que presentaron IP, el $36,4 \% \quad(n=32)$ era portador mientras que de los 83 que no tuvieron IP eran portadores el 31,3\% $(n=26)$, no encontrándose diferencias significativas entre los grupo $(p=0,487)$. Destacar que $15,3 \%(n=31)$ de casos perdidos.

\section{Causas de retirada}

El 85,89\% ( $n=207)$ de los pacientes tuvo un catéter mientras estuvo en DP, el 12,03\% ( $n=29)$ tuvo 2 catéteres y el $2,07 \%(n=5)$ tuvo 3 catéteres.

Las causas de retirada de los catéteres se describen en la tabla 4. Tras la salida de DP (trasplante, exitus o paso a hemodiálisis) que supuso un $45,6 \%$ de las retiradas de catéter, el $10 \%$ se debió a una IP y el $6,6 \%$ a un mal funcionamiento del catéter. El autoposicionante se retiró en más casos por fuga, infecciones peritoneales y mal funcionamiento. 


\begin{tabular}{|l|c|c|c|}
\hline & \multicolumn{2}{|c|}{ Tipo de Catéter } & Total \\
\hline & $\begin{array}{l}\text { Catéteres } \\
\text { Tenckhoff }\end{array}$ & Autoposicionante & \\
\hline Fuga & $0 \%$ & $6,5 \%(n=10)$ & $\begin{array}{c}4,1 \% \\
(n=10)\end{array}$ \\
\hline $\begin{array}{l}\text { Infección } \\
\text { Túnel }\end{array}$ & $3,4 \%(n=3)$ & $0 \%$ & $\begin{array}{c}1,2 \% \\
(n=3)\end{array}$ \\
\hline $\begin{array}{l}\text { Infección } \\
\text { Peritoneal }\end{array}$ & $8 \%(n=7)$ & $11,1 \%(n=17)$ & $\begin{array}{c}10 \% \\
(n=24)\end{array}$ \\
\hline $\begin{array}{l}\text { Mal } \\
\text { funcionamiento }\end{array}$ & $3,4 \%(n=3)$ & $8,5 \%(n=13)$ & $\begin{array}{c}6,6 \% \\
(n=16)\end{array}$ \\
\hline $\begin{array}{l}\text { Salida de } \\
\text { Diálisis } \\
\text { Peritoneal }\end{array}$ & $\begin{array}{l}52,3 \% \\
(n=46)\end{array}$ & $41,8 \%(n=64)$ & $\begin{array}{r}45,6 \% \\
(n=110)\end{array}$ \\
\hline Funcionante & $\begin{array}{l}33 \% \\
(n=39)\end{array}$ & $32 \%(n=49)$ & $\begin{array}{l}32,4 \% \\
(n=78)\end{array}$ \\
\hline
\end{tabular}

Tabla 4. Causas de retirada del Catéter Peritoneal

\section{Supervivencia de los catéteres}

También analizamos la supervivencia de ambos tipos de catéteres (Figura 1). No hallamos diferencias en la supervivencias de los Catéteres Tenckhoff respecto a los autoposicionante (Log-Rank Mantel Cox =0,164). La media de supervivencia de los catéteres Tenckhoff fue de 1116,33 (IC95\%: 907,12-1325,54) frente a 942,128 (IC $95 \%$ : 800,4-1083,85) días de los autoposicionante.

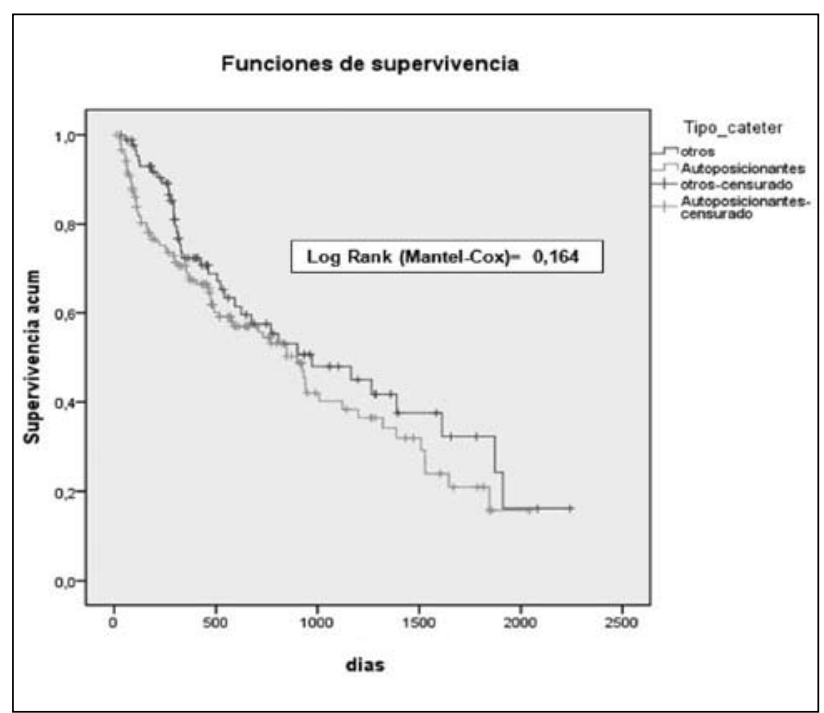

Figura 1. Supervivencia de los dos tipos de catéteres de Diálisis peritoneal
Mediante una análisis de regresión estudiamos la edad, las infecciones peritoneales y la fuga como variables que pudieran influir sobre la supervivencia del catéter, encontrando significación $(p=0,007)$ para la edad HR 1,017 (IC95\%: 1,005-1,030) y la fugas $(p<0,001)$, HR 2,026 (IC95\%: 2,026-6,83).

Mediante una análisis de regresión estudiamos la edad, las infecciones peritoneales y la fuga como variables que pudieran influir sobre la supervivencia del catéter, encontrando significación $(p=0,007)$ para la edad HR 1,017 (IC $95 \%$ : 1,005-1,030) y la fugas $(p<0,001)$, HR 2,026 (IC95\%: 2,026-6,83).

\section{Salida de DP}

De los pacientes estudiados permanecen en activo en nuestro programa de DP el $37,1 \%(n=75)$, siendo la principal causa de salida el exitus $24,3 \%(n=49)$, seguido del trasplante renal $18,8 \%(n=38)$.

El $15,3 \% \quad(n=31)$ de los pacientes pasaron a hemodiálisis por otras razones (decisión personal, fugas, cirugía abdominal...) y el $1,5 \%(n=3)$ recuperaron la función renal, pasando a la consulta de enfermedad renal crónica avanzada. Sólo el 3\% $(n=6)$ tuvo que abandonar la técnica por una IP.

\section{Discusión}

Muchos autores han descrito las características ideales que debería poseer el catéter peritoneal ideal y se han diseñados muchas variantes del mismo (TWH, SwanNeck, Cruz, etc) ${ }^{1}$, pero ninguno le ha dado un solución definitiva al problema del acceso peritoneal.

Actualmente, los catéteres mas utilizados son los Tenckhoff ${ }^{3-4}$ aunque varia según las comunidades ${ }^{3}$. Estos catéteres presentan disfunciones que suelen estar relacionadas con el desplazamiento de la punta del mismo ${ }^{5}$, por lo que en 1996, Di Paolo y col. diseñaron el catéter autoposicionante que debía reducir estas complicaciones gracia a su diseño².

El catéter más utilizado en nuestra muestra fue el autoposicionante debido a la diferencia entre las poblaciones que atienden nuestras unidades. De igual forma, y aunque nuestros registros tienen una mayor amplitud, el estudio se ha limitado a 5 años de seguimiento, coincidiendo 
con el comienzo del uso del catéter autoposicionante en una de las unidades de D.P, tras los buenos resultados comunicado por diversos autores s-6-7-8. $^{2}$.

El éxito de los programas de DP depende al menos en parte, de una adecuada colocación de los catéteres y la ausencia de complicaciones quirúrgicas tras su implantación. Las complicaciones quirúrgicas descritas en los catéteres autoposicionantes pueden deberse quizás a no contar con un equipo fijo para la implantación del catéter peritoneal en el centro que los utiliza'. Varios autores han descrito la mayor incidencia de fugas y perforaciones viscerales con el catéter autoposicionante debido a su diseño ${ }^{5}$, y lo comprobamos al analizar las complicaciones mecánicas donde a pesar de que sólo fue significativa la fuga, todas fueron más frecuentes excepto el desplazamiento. También podría deberse a que el lastre del catéter puede favorecer una mala cicatrización y fugas pericatéter cuando no ha habido una buena fijación del trayecto intraparietal y un reposo relativo del paciente los primeros días tras la implantación ${ }^{4}$. Curiosamente, también se ha descrito en la literatura picaduras del segmento inter-cuffs en los catéteres autoposicionantes que pueden deberse a problemas de manufacturación puntual ${ }^{10}$.

No encontramos diferencias significativas al analizar la aparición de IP en función del catéter utilizado, ya que ambos catéteres disponen de un doble cuff y ambas unidades siguen los mismos protocolos de cuidados. De igual manera tampoco se han encontrado diferencias entre los portadores de estafilococos aureus y los no portadores ya que desde que se implanto el protocolo de cribado de los estafilococos aureus, y su tratamiento con Mupirocina, hemos reducido las tasas de IP en los mismos hasta igualarlas ${ }^{11}$.

También analizamos la supervivencia de ambos tipos de catéteres y hallamos una mayor supervivencia de los catéteres Tenckhoff respecto a los autoposicionante desde el inicio del estudio y que se mantienen a lo largo del mismo, aunque sin significación estadística. Debido a los resultados que presentamos, parece deberse a las fugas halladas en el grupo de los catéteres autoposicionantes, como vemos en el modelo multivariante, y que tienen lugar en el momento de su implantación.

El $85 \%$ de los pacientes solo necesita un catéter peritoneal a lo largo del tratamiento. Sin embargo al analizar las causas de retirada de los catéteres, la fuga y la disfunción del catéter se dan más en los autoposicionantes.

Como conclusión, y en este estudio, los catéteres autoposicionante han tenido un mayor número de complicaciones quirúrgicas, un mayor número de complicaciones mecánicas que los Tenckhoff, sin encontrar diferencias respecto a las complicaciones infecciosas.

Aunque sin significación estadística, también hemos haIlado una menor supervivencia respecto a los Tenckhoff. Todos estos datos parecen estar relacionados con las fugas producidas por los catéteres autoposicionantes desde su implantación.

Recibido: 1 Noviembre 2012

Revisado: 8 Noviembre 2012

Modificado: 12 Noviembre 2012

Aceptado: 13 Noviembre 2012

\section{Bibliografía}

1. Martín Espejo, J.L. Catéteres peritoneales. Tipos de catéteres. Cuidados del catéter pre y post implantación según protocolo del grupo de D.P. Andalucía. Complicaciones inmediatas después de la colocación del catéter. En: VII Curso de D.P. para Enfermería Nefrológica de Andalucía.2011. [acceso 20 0ctubre 2012]. p 67-81. Disponible: http://www.revistaseden.org/files/3062_cateter\%20peri.pdf.

2. Di Paolo N, Sansoni E, Cappelletti F, et al. The self-locating catheter- review and analysis of costs. The international journal of artificial organs. 2006; 29(1):00-00.

3. Remón $\mathrm{C}$, Quiros $\mathrm{PL}$, Perez-Bañasco V, Torán $\mathrm{D}$, Tejuca $F$, Merino $M J$, et al. Informe del registro de pacientes con insuficiencia renal crónica en tratamiento con diálisis peritoneal de Andalucía: periodo 1999-2004. Nefrología 2006; 26:45-55.

4. Dombros N, Dratwa M, Feriani M, et al. EBPG Expert group on Peritoneal Dialysis. European best 
practice guidelines for peritoneal dialysis. 3 peritoneal access. Nephrol Dial Trasplant 2005; 20 (suppl 9):s8-12.

5. Lanuza, M, Minguela JI, Rodado R, et al. Our nineyear experience with the self-locating catheter: comparision of malfunction rate with other Tenckhoff catheter variants. The international journal of artificial organs. 2006; 29(1):138-141.

6. De Alarcón, R.M, Roca, S, Gimeno, C. et al. Catéter Autoposicionante. Experiencia clínica en nuestra unidad. En: Libro de comunicaciones de la VIII Reunión Nacional de D.P; Oviedo 2-4 de febrero 2012. Fresenius Medical Care; 2012. p 134.

7. Lanuza, M., Minguela, J.I., Martínez-Loza, A. y Col. Mínimas complicaciones mecánicas con el catéter autoposicionante. ¿Es posible un solo catéter para cada paciente en D.P.? En: Libro de comunicaciones de la VIII Reunión Nacional de D.P; Oviedo 2-4 de febrero 2012. Fresenius Medical Care; 2012. p 135.

8. Sánchez Canel, J.J, García Pérez, H, Aicart, C. et al. Ensayo prospectivo y randomizado para la com- paración de catéteres Autoposicionantes y Tenckhoff recto en pacientes en D.P. En: Libro de comunicaciones de la VIII Reunión Nacional de D.P; Oviedo 2-4 de febrero 2012. Fresenius Medical Care; 2012. p 35-37.

9. Sanchez, J.E., Rodriguez, C., González, I. y Col. Las complicaciones precoces tras la inserción del catéter predicen la aparición de la infección peritoneal y se relacionan con los cambios en el equipo quirúrgico. En: Libro de comunicaciones de la VIII Reunión Nacional de D.P; Oviedo 2-4 de febrero 2012. Fresenius Medical Care; 2012. p 136.

10. Moreiras Plaza, M., Nájera de la Garza, W., Blanco García, R. et al. Nuevos catéteres: ¿Nuevos problemas? En: Libro de comunicaciones de la VIII Reunión Nacional de D.P; Oviedo 2-4 de febrero 2012. Fresenius Medical Care; 2012. p 132.

11. Gómez A. C., Sánchez J. Portadores nasales de estafilococo áureo en diálisis peritoneal: infecciones del orificio. En: Libro de comunicaciones de la XXVII Congreso de la Sociedad Española de Enfermería Nefrológica; Bilbao. 16-19 de 0ctubre 2002. HOSPAL; 2002. p 152-159. 